Volume No. 1, Issue No. 3 (July-September), pp. 15-25

\title{
Cognitive Experiments and Features for Computing Mental Stress
}

Khalid Masood , Haroon Ur Rashid

\begin{abstract}
In this paper, mental stress is computed through cognitive experiments that induce stress. In a controlled laboratory environment, a group of students are involved in a series of mental challenges. While performing the cognitive tasks, stress is induced on the participants. Deep breathing exercise is performed in the start of experiments and then in between each activity to make the conditions normal and a participant feels relaxed. Various physiological features are recorded during experimental activities. Also, cerebral features are recorded that provide improved classification results. The severity of stress is different on each participant but the purpose of experimental protocol is to separate stressful conditions from relaxed environment. Support Vector machine (SVM) is used to identify relax or normal class from a number of stressed classes. It is shown that cerebral features improve the classification accuracy with a satisfactory margin and designed protocol system is able to compute the severity of induced stress.
\end{abstract}

Key words - Mental Stress, Cognitive experiments, Cerebral Features, Heart rate variations, Electrodermal Activity, Support Vector Machine.

\section{INTRODUCTION}

Stress is ever prevalent in modern society. Chronic or long-term stress has both psychological and physiological effects. Several diseases such as diabetes, insomnia hypertension and depression can be caused by chronic stress [1]. Also a few social problems including family quarrels, marriage nullation, violence, suicides and ${ }^{3}$ anger on streets and roads can be increased due to stress. To accurately diagnose chronic stress, physicians may want to observe a stressful person for long durations.

${ }^{1}$ Khalid Masood , ${ }^{2}$ Haroon Ur Rashid

${ }^{1}$ University of Jeddah, ${ }^{2}$ Lahore Garrison University 
As stress is a psychological reaction between outer demands and environmental inputs that cross the barrier of a human capacity to sustain pressure [2], personal state monitoring includes not only monitoring the physiological reactions to stress but also the perceived psychological impacts of the stressors. Assessment of the physiological impact can be reported with limited wearable sensors that record physiological signals including changes in heart rate, variations in respiration rate and changes in conductance of the skin. The psychological impact is more difficult to ascertain and requires wearable sensors to monitor daily stress levels in a person's busy and routine life.

As each individual has different feel and capacity for stress, the impact of stress is different on each person. The physiological responses occur due to hormones from the pituitary glands and brain hemispheres, whose level is highly dependent on a person's psychological conditions and his reaction to the imposed situation [3] thus varying their severity or scale of stress.

To design a protocol to record stress, the quality metrics should not only incorporate a person's psychological conditions but also consider a few physiological variations inside a human body. This complicates the development of a general system that can measure stress because for each person, tuning should be performed to incorporate his mental maturity.

An accurate stress monitoring system thus should monitor not only monitor not only physiological impacts but psychological effects should also be considered so that a system is designed that fully captures all stress related effects. The stress monitoring system should balance between psychological and physiological conditions of a person. Hence, we have developed a generalized stress measurement system that monitors a person's physiological parameters and adjusts them to his/her psychological conditions and a fine tuning can be performed for each individual. Assessment for both physiological and psychological impact of stress on a person needs to be performed and translate these assessments into an objective quality metric that is valuable to physicians.

The adapted scores are then used in a pattern classification algorithm as 'ground-truth' stress levels to be predicted from wearable sensor signals. The result is thus an accurate estimate for the severity of stress as felt by an individual based on both their physiological and psychological responses. Literature review is presented in section 2. In section 3, we present the methodology adopted to collect data used in the development of the adaptive model. The design and implementation details to collect 
the features are provided in section 4. In section 5, advantages of proposed model and results are explained. Conclusions from this study and future work are presented in section 6 .

\section{LITERATURE REVIEW}

An individual reacts to stress according to his/her own self-esteem and strength of mind. For a unique situation, different people react differently. The feel of stress is different in each person depending upon his/her inner control over his/her emotions and its responses. The people who are more confident and have high self-esteem feel less stress than the people who get more stressed. To interpret a subject's perception of the level of severity of stress, selfreporting questionnaires are used. Selfreporting scores (SRS) are a set of scales, used to measure the conventionally defined negative or positive emotional states that represent anxiety and stress [4]. These scores were developed to identify individuals who meet essential criteria for a specific level of stress and anxiety.

However, there is a need to validate the accuracy and efficiency of the self-reported scores as the importance of scores is significant for stress related symptoms (self-reported scores provide opportunities for early intervention of people at risk).

The self-reported scores are used in a number of studies to correlate stress with physiological parameters. Three samples of student population were chosen based on an anxiety scale of depression. It was reported that disturbance in sleep, fatigue and constipation were more common in depressed people than healthy persons. Clinical depression and spoiled moods tend to increase the symptoms of depressive symptomatology and cortisol levels. In another study, 36 students who are nonsmoking took participation to evaluate selfreported scores. Using heart rate variability (HRV) analysis, it was reported that there is a positive correlation between stress and low frequency power waves of heart signals. Using two samples of female participants containing 36 stress related depressed women and 19 women who are healthy, it was reported that the first group has low values of HRV, high in temperatures and $\mathrm{O}_{2}$ saturation at fingers is lower than the second group who is healthy stress [5]. Chronic stress causes permanent and long-term variations in people with stress which is evident from their stress response inventory (SRI) scores. To test the validity of perceived stress scale questionnaire (PSQ), statistical testing was experimented on a group of participants. It was reported that work discontent and work pressures are the main causes for increase in their stress response inventory (SRI) 
scores [6].

Though self-reporting scores are beneficial due to their low cost, efficient analysis and ability to collect larger amounts of data, concerns have been expressed about the reliability of the extracted self-report measures [7]. Their robustness and accuracy can be disputed as self-reports are always more doubtful than indirect or direct observations [8]. Generally, there are two types of socially desirable responses, impression management and selfdescription. Changes or variations in impression facilitates favorable tendency to provide biased comments and hence is a form of deception and falsification. Selfdescription can be defined as it is a positive opinion but it is subjective and depends on various factors. In such cases, a participant who scores high on self-description is certain that his/her positive opinions are true. Subjects tend to report what reflects positively on their own abilities. In general, it is advised that variations in impression and self-description scores should be thoroughly checked and verified by alternate methods such as performing objective analysis of the data [9]. To test the validity of self-reported scores, we have designed and developed an adaptive model that minimizes these deviations in stress scores. An individual's self-reported score is based on his physiological state and his perception of the situation. In a negative psychological mood or due to wrong perceptional judgment, an error or deviation is introduced in his score [10]. Our adaptive model not only computes deviations in self-reported scores but provides tuning which incorporates into the system an individual's physiological variations and adjusts them accordingly to provide a generalized solution for determining their level of mental stress.

\section{METHODS}

\subsection{Stress Monitoring System}

It is recommended that a wearable system that is used for long term should not only be relaxing but also flexible with accurate results so that long term monitoring of a potential subject can be performed. A few physiological parameters containing blood pressure and blood flow are not suited for long term monitoring. Instead variations in heart rate can be used to identify and compute stress. As stress increases, variations in heart rate decreases. Another measure that is used frequently to determine stress is electro dermal activity (EDA).

It varies in proportion with stress levels as with increase in stress, the EDA increases due to increase in conductivity of the body with perspiration and sweating. Respiration patterns can also be used to measure stress. 
Breathing pattern is found to be irregular and respiration rate increases when a person is under stress. It is reported that EDA, HRV and respiration rate are more suitable physiological parameters that can be used to determine stress for long term observations as they can be monitored without obstructing day to day activities. Information content and accuracy of sensors is less affected using these measures and overall comfort can be achieved with long durations of wearability [11].

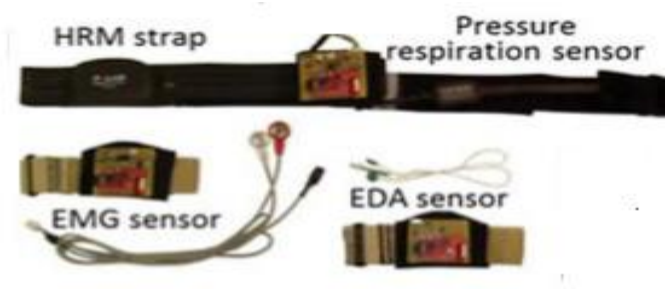

Figure 1: Hardware components containing HRM, $E D A$ and Pressure respiration sensors.

It is in this context that we have designed a wearable wireless sensor system that records respiration patterns, heart rate variations and electro dermal activity where details of the system are described in Figure 1. To measure heart rate variability, the system uses a heart rate monitor from Polar Electro Inc. For respiration, the sensor SA 9311M, made by Thought Technology Limited, is integrated into the HRM strap.
Electro dermal (EDA) activity is measured with two $\mathrm{AgCl}$ electrodes (Vivo metrics Systems Corp.) on the index and middle fingers. The sensor network transmits data to a holster unit that contains a sensor hub along with a data processor and a battery made of lithium polymer that can provide 15 hours continuous operation with storing data onto a micro SD card.

\subsection{Experimental Protocol}

There were 22 students (age bracket: 18-30, male: 12, female: 10) who participated in the stress related experiments that are approved by IRB (Institutional Review Board) with the contract number as ER-99186. To develop an accurate metric that combines the psychological and physiological impact of stress on a person, we collected data using our wireless wearable platform, while subjects performed a series of cognitive activities, with varying levels of stress induction depicted by recorded physiological parameters. To compute stress levels, the subjects were also asked to fill out three surveys, first is stress surveys followed by difficulty surveys that is completed after completion of each challenge and a post stress survey, immediately after completion of all activities. In these surveys, subjects were asked to self-score each activity on a 7-point scale that is called Likert scale in 
which 1 represents no stress conditions while 7 represents extreme stressful conditions. At the start of the experiments, participants were instructed to undergo a 3mint deep breathing exercise $(0.1 \mathrm{~Hz}$ breathing rate) to help bring them to a relaxed state. Deep breathing was also used between stressful activities to aid in recovery. The cognitive activities include a dual tracking task where a participant has to track a varying object using a mouse and a screen where a key letter is displayed. In memory search exercise, participants have to remember a set of words and later they are asked to reproduce it using various confounders. In mirror tracing challenge, participants have to trace a pattern by looking onto the reflection in a mirror. In the next challenging activity of strop test, words are displayed in different ink colors and participants are required to click the result depending on sound or ink color of the word. In supine and tilt positions, physiological parameters are recorded and finally in public speech task, participants have to perform a public speech of 4-minute duration). Figure 2 below gives the sequence of activities in the experiment.

\section{FEATURE SET}

To provide medical practitioners with an accurate assessment of the personal state of an individual, we have developed an adaptive model that compares recorded physiological measures with self-reported scores to identify deviations in either of these two categories. Deviations identified in the physiological measures are adapted using a weighting factor scheme based on the median value of the features obtained from all the subjects. Deviations computed in the self-reported scores are used to identify unreliable rankings made by the subjects, which are then excluded from the data set. By identifying these deviations, the adaptive model provides a data set which more accurately reflects the subjects' physiological state enabling a reliable estimate of their stress levels. During the experiments outlined in Figure 2, we determined HRV, EDA and respiration patterns, while subjects performed the specified activities. The SCL (skin conductance level) and SCR (skin conductance responses) in EDA were computed using a regression based model on least-squares detrending method. In this approach, SCL is represented by aperiodic response and SCR is represented by the residual signal. We have computed four features, two each from SCL and SCR that are represented by mean and standard deviation of the signal as follows,

$\mu_{S L}=\frac{1}{N} \sum_{i=1}^{N} R_{S L}(t-i)$ 
Where $\mu_{S L}$ is the average SCL trend for N samples of aperiodic signal?

The standard deviation is computed as follows

$\sigma_{S L}=\left[\frac{1}{N} \sum_{i=1}^{N} R_{S L}(t-i)\right]^{1 / 2}$

Where $\sigma_{S L}$ is the average SCL trend for $\mathrm{N}$ samples of aperiodic signal?

Similarly, $\mu_{S R}$ and $\sigma_{S R}$ are derived using transformation in residual SCR.

The feature vector consists of various physiological parameters containing low and high frequency power ranges and using successive RR intervals, mean and standard deviations are computed. Another feature is PNN15 that is computed using the portion of RR interval which is not varied in more than $15 \mathrm{msec}$. Finally, the root mean square of power density signal is computed using consecutive differences of RR sample. To compute cerebral features, EEG signals are computed from two electrodes that are attached to right and left hemisphere of a person. A total number of four cerebral

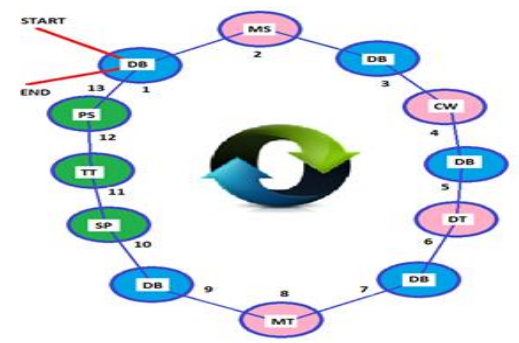

Figure 2: Cognitive Activities showing Deep breathing exercise in between each activity. features are calculated and classification is performed with and without cerebral features.

\section{RESULTS}

We have performed classification using support vector machine (SVM) classification model with the goal of predicting stress state of the subjects based on the extracted physiological features of HRV and EDA and their self-reported scores. As the relaxation activity in the protocol was deep breathing, where subjects controlled their respiratory rate, it was not used in the classifier to avoid biasing of the results. The output of the classifier was a metric which classified the mental state of the individual as being in either a relaxed class (0), low stress class (1) or high stress class (2). The input to the model was in the form of recorded physiological parameters which are mapped or labeled to three mental states or classes as provided by user's self-reported scores.

In our supervised model, stress scores are used for labels of the classes. Based on an initial k-means clustering analysis, we employed different number of clusters and found that compactness and separation of data is better in three clusters than four or five clusters. Hence, the score labels were divided into three classes as follows, 
- Stress score 1 represents Class 1 (Relax class)

- Stress score 2, 3 \& 4 denote Class 2 (Low stress class)

- Stress score 5, $6 \& 7$ represent Class 3 (High stress class)

Initial analysis indicated that the separation between three classes is better in EDA than HRV features. In HRV, the high stress group is well separated but low stress and relax groups are intermixed. The heart rate variation is high during deep breathing activities but becomes low in stressful conditions thus allowing the stress group to be discriminated from the rest. HRV and EDA features are used for classification. As seen the three clusters, relax, low stress and high stress groups are well separated from each other.

A SVM was chosen as it performs a nonlinear projection of input variables onto high dimensional feature space such that features are segregated in a pattern that a linear boundary can be used to separate the corresponding clusters. In this case the SVM was used to map physiological parameters into self-reported scores. The SVM was based upon a radial basis function (RBF) kernel, with the optimum selection of cost function $\mathrm{C}$ and error value $\gamma$ using cross validation testing. During cross validation, tuning based on a grid search technique is performed such that different pairs of $(\mathrm{C}, \gamma)$ are iterated and that pair who has the highest accuracy, is chosen for training experiments.

Two third of samples were used in testing sample and one third remaining samples are used for training. Using five-fold cross validation, the data is employed by dividing training samples into five equal sets. SVM was trained on four sets of data and fifth set was used in testing. The tuning was applied to the training set using a sequential grid search. In this procedure, an iterative algorithm is applied for the incremental values of cost of optimization and bandwidth $\gamma$. The optimum values for $\gamma$ is found to be 0.1 while the cost function is computed as 0.125 after the tuning.

\section{CONCLUSIONS}

We have designed an adaptive model which has two folded effect on the system. First, it contains adjustable and tuning parameters which can adapt themselves to the individual. Secondly, it accurately classifies an individual's personal state based on physiological and psychological measures. Thus, a generalized model for computation of stress is proposed which can adjust to the user's individual inner state. The initial classification error of $24 \%$ is reduced to 10 $\%$ using our adaptive algorithm in two steps. First, tuning is performed which improves accuracy by $6 \%$. Secondly, 
subjective deviations are subtracted from the classification error to obtain $85 \%$ classification accuracy for the adaptive model, as shown in Figure 3 and Table 1. As stress or intensity of the reaction is in proportion to one's ability to cope with danger and degree of control over emotions, there is a need for a model which can be adjusted to levels of reactions (of stress) which vary from person to person. The proposed adapted model fulfills this requirement by using a wearable wireless sensors system that can be worn for long durations with ambulatory monitoring of a number of physiological parameters using self-corrections and auto adjustments. In future, the next immediate step is to perform experiments on bigger data sets collected over longer periods of time to validate the proposed adaptive model. We also plan to investigate effect of stress on gender. Finally, methods to tune the selfreported scores, including stress and anxiety baseline surveys will be pursued.

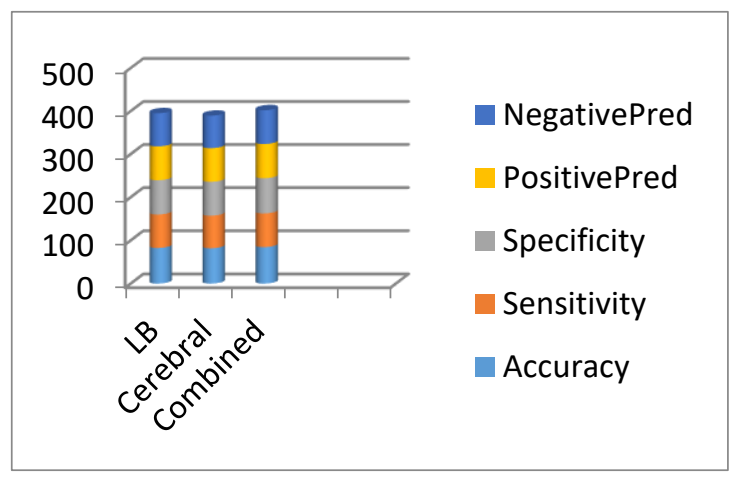

Table 1: Quality Metrics and Classification Results

\begin{tabular}{|l|l|l|l|}
\hline \multicolumn{1}{|c|}{ Quality Metrics } & Lower body & Cerebral & Combined \\
\hline Accuracy & 82.5 & 81.8 & 84.5 \\
\hline Sensitivity & 77.5 & 75.5 & 78 \\
\hline Specificity & 79.5 & 78.5 & 81.5 \\
\hline Positive PredValue & 78.5 & 78 & 79.5 \\
\hline Negative PredVaue & 77 & 75.5 & 78.5 \\
\hline
\end{tabular}




\section{REFERENCES}

[1] C. Setz, B. Amrich, J. Schumn, R. Marca, G. Trster and U. Ethlert, "Discriminating stress from cognitive load using a wearable EDA device," IEEE Transactions on Information Technology and Biomedicine, vol. 14, no. 2, pp. 410417, 2010.

[2] B. Ahmed, H. Khan, J. Choi and R. Guiterez-Osuna, "ReBreathe: A calibration protocol that improves stress/relax classification to relabelling deep breathing relaxation exercises," IEEE Transactions on Affective Computing, vol. 7, pp. 150161, 2016.

[3] L. Bouarfa, P. Bembnowicz, B. Crewther, D. Jarchi and G. Z. Yang, "Profiling visual and verbal stress responses using electrodermal heart rate and hormonal measures," IEEE International Conference on Body Sensor Networks (BSN), pp. 1-7, 2013.

[4] C. Tsigos and G. P. Chrousos, "Hypothalamic-pituary-adernal axis neuroendocrine factors and stress," Journal of Psychosomatic Research, vol. 53, pp. 865-871, 2002.
[5] J. Healey and R. Picard, "Smartcar: detecting driver stress," Proceedings of 15th International Pattern Recognition Conference, vol. 4, pp. 218-221, 2000.

[6] M. H. Lee, G. Yang, H. K. Lee and S. Bang, "Developmental stress monitoring system based on personal digital assistant (PDA)," IEEE Confernce on Engineering Medicine and Biology Society, vol. 4, pp. 2364-2367, 2004.

[7] J. Zhai, A. B. Barreto, C. Chin and C. Li, "Realization of stress detection using psychophysiological signals for improvement of human computer interactions," IEEE Confernce on Southeast, pp. 415420, 2005.

[8] B. Grundlehner, L. Brown, J. Penders and B. Gyselincks, "The design and analysis of a real time, continuous arousal monitor," Proceedings of 6th International Workshop and Implantable Body Sensor Networks (BSN), pp. 156161, 2009.

[9] H. Labbe, "Coping with stress, the effectivenes of different types of music," Applied Psychophysiology 
and Bio feedback, vol. 32, pp. 163168, 2007.

[10] J. Thayer, S. Yamamoto and J. Brosschot, "The relationship of autonomic imbalance, heart rate variability and cardiovascular disease risk factors," International Journal of Cardiology, vol. 141, no. 2, pp. 122-131, 2010.

[11] Q. Wu, Y. Xu, S. Feng, Z. Du and J. Wang, "Cognitive Internet of THings: A New Paradigm beyond Connection," IEEE, 2014. 